\title{
Fractional Change in Apparent Diffusion Coefficient as an Imaging Biomarker for Predicting Treatment Response in Head and Neck Cancer Treated with Chemoradiotherapy
}

\author{
M. Matoba, H. Tuji, Y. Shimode, I. Toyoda, Y. Kuginuki , K. Miwa, and H. Tonami
}

\begin{abstract}
BACKGROUND AND PURPOSE: ADC provides a measure of water molecule diffusion in tissue. The aim of this study was to evaluate whether the fractional change in ADC during therapy can be used as a valid predictive indicator of treatment response in head and neck squamous cell carcinoma treated with chemoradiotherapy.
\end{abstract}

MATERIALS AND METHODS: Forty patients underwent DWI at pretreatment and 3 weeks after the start of treatment. The pretreatment $A D C$, fractional change in $A D C$, tumor regression rate, and other clinical variables were compared with locoregional control and locoregional failure and were analyzed by using logistic regression analysis and receiver operating characteristic analysis. Furthermore, progression-free survival curves divided by the corresponding threshold value were compared by means of the log-rank test.

RESULTS: The fractional change in $A D C_{\text {primary }}$, the fractional change in $A D C_{\text {node }}$, primary tumor volume, nodal volume, tumor regression rate $_{\text {node, }} \mathrm{N}$ stage, and tumor location revealed significant differences between locoregional failure and locoregional control $(P<.05)$. In univariate analysis, the fractional change in $A D C_{\text {primary }}$, fractional change in $A D C_{\text {node }}$, tumor regression rate ${ }_{\text {node }}, N$ stage, and tumor location showed significant association with locoregional control $(P<.05)$. In multivariate analysis, however, only the fractional change in $A D C_{\text {primary }}$ was identified as a significant and independent predictor of locoregional control $(P=.04)$. A threshold fractional change in $A D C_{\text {primary }}$ of 0.24 revealed a sensitivity of $100 \%$, specificity of $78.7 \%$, and overall accuracy of $84.8 \%$ for the prediction of locoregional control. Progression-free survival of the 2 groups divided by the fractional change in $A D C_{\text {primary }}$ at 0.24 showed a significant difference $(P<.05)$.

CONCLUSIONS: The results suggest that the fractional change in $A D C_{\text {primary }}$ is a valid imaging biomarker for predicting treatment response in head and neck squamous cell carcinoma treated with chemoradiotherapy.

ABBREVIATIONS: $\triangle A D C=$ fractional change in $A D C ; H N S C C=$ head and neck squamous cell carcinoma; $\triangle T V=$ tumor regression rate; $L R C=$ locoregional control; $\mathrm{LRF}=$ locoregional failure

A pproximately two-thirds of patients with head and neck squamous cell carcinoma (HNSCC) present with advancedstage disease, and regional lymph node involvement is common. ${ }^{1}$ Surgery with or without adjuvant chemotherapy and/or radiation therapy remains a mainstay of treatment in advanced HNSCC, but radical radiation therapy alone or concurrent chemoradiotherapy as a definitive treatment has become a standard management option for many patients with HNSCC to improve the pa-

Received February 17, 2013; accepted after revision May 17.

From the Departments of Radiology (M.M., I.T., Y.K., H. Tonami) and Otorhinolaryngology (H. Tuji, Y.S., K.M.), Kanazawa Medical University, Ishikawa, Japan.

Please address correspondence to Munetaka Matoba, MD, Department of Radiology, Kanazawa Medical University, Daigaku 1-one, Uchinada, Kahoku, Ishikawa, 9200293, Japan; e-mail: m-matoba@kanazawa-med.ac.jp

http://dx.doi.org/10.3174/ajnr.A3706 tient's quality of life via organ preservation. Despite these rigorous treatment methods, however, locoregional disease failure occurs in as many as $30 \%-40 \%$ of cases. ${ }^{2,3}$ Therefore, if a reliable indicator of response to radiation therapy or chemoradiotherapy before or at an early stage of treatment could be found, patients whose prognoses are likely to be unfavorable with current approaches might be selected for alternative strategies, improving their chances of success and sparing them from ineffective treatment with unnecessary toxicity. It has been impossible, however, to reliably predict early individual treatment response despite careful evaluation by using traditional clinical predictors such as tumor size, clinical stage, tumor location, and lymph node involvement. ${ }^{4}$

DWI extracts information from the diffusion of water molecules in tissue. Water molecule diffusion motion can be quantified by using the ADC. In general, highly cellular cancers have more restricted diffusion, resulting in lower ADC values, while 
Histologically confirmed HNSCC between January 2008 and September 2012

Inclusion criteria:

- No previous treatment history (primary case)

- Tumor volume and site appropriate to chemoradiotherapy with curative intent

- No history of radiotherapy in the head and neck region

- Performance status of 0-1 (Eastern Cooperative Oncology Group scale)

- Age $\leqq 80$ years

- Leukocyte $\geqq 4000 / \mu \mathrm{L}$, platelets $\geqq 100,000 / / \mu \mathrm{L}$, hemoglobin $\geqq 9.5 \mathrm{~g} / \mathrm{dL}$ serum creatinine $\leqq$ normal institutional upper limit, 24 -hour creatinine clearance $\geqq 60 \mathrm{~mL} / \mathrm{min}$ bilirubin $\leqq 1.5 \mathrm{mg} / \mathrm{dL}$, asparate aminotransferase and alanine transferase $\leqq$ normal upper limit

Exclusion criteria:

- Active invasive malignancies in the 3 years leading up to protocol entry

- Distant metastases

- Serious complications: active infectious disease, interstitial pneumonia, cardiac failure, renal failure, liver dysfunction, etc

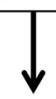

40 consecutive patients for predicting the treatment response has not been established, to our knowledge.

The aim of this study was to evaluate the usefulness of the fractional change in $\mathrm{ADC}(\triangle \mathrm{ADC})$ during therapy for prediction of treatment response in patients with HNSCC treated with chemoradiotherapy compared with the other clinical variables and to identify whether the $\triangle \mathrm{ADC}$ during therapy can be used as a valid imaging biomarker for prediction of treatment response.

\section{MATERIALS AND METHODS Patient Population}

This prospective study was approved by the Committee on Clinical Study at our institution, and written informed consent was obtained from all patients. The study population consisted of patients with histologically confirmed primary HNSCC who were treated with chemoradiotherapy between January 2008 and September

FIG 1. Patient selection criteria.

Table 1: Patient characteristics

\begin{tabular}{lc}
\hline \multicolumn{1}{c}{ Characteristics } & No. of Patients $(\boldsymbol{n}=\mathbf{3 5})$ \\
\hline Age (yr) & \\
Median & 66.2 \\
Range & $33-79$ \\
Male/female & $30: 5$ \\
Tumor location & \\
Supraglottis & 3 \\
Oropharynx & 9 \\
Hypopharynx & 9 \\
Larynx & 10 \\
Oral cavity & 4 \\
Tstage (UICC 2002) & \\
T1 & 1 \\
T2 & 14 \\
T3 & 8 \\
T4 & 12 \\
N stage (UICC 2002) & \\
N0 & 3 \\
N1 & 7 \\
N2 & 20 \\
N3 & 5 \\
\hline
\end{tabular}

Note:- UICC indicates Union for International Cancer Control.

cancer treatments causing cell death increase water diffusion and lead to a rise in $\mathrm{ADC}^{5}$

In HNSCC, recent clinical studies have applied DWI to the prediction of treatment response to neoadjuvant chemotherapy, radiation therapy, or chemoradiotherapy before or at an early stage of treatment, revealing that pretreatment ADC correlates with treatment response and that $\mathrm{ADC}$ changes at 1,2 , and 4 weeks after the start of treatment can predict treatment response. ${ }^{6-9}$ In addition, it has been reported that ADC changes at 3 weeks posttreatment can predict treatment response with higher accuracy than morphologic imaging assessment. ${ }^{10}$ Studies evaluating the predictive value of DWI for treatment response post-radiation therapy and/or chemotherapy are limited, however, and the optimal timing of the evaluation of the DWI and ADC analysis method
2012 at our institution. Patient selection was performed according to the inclusion and exclusion criteria for this study, summarized in Fig 1. Forty patients who met these criteria were enrolled in this study. Five patients were excluded from the data analysis: 2 who refused the proposed treatment, 2 for whom the MR image quality was poor due to a low signal-to-noise ratio or artifacts, and 1 who died within 3 months after therapy with unknown disease status. Eventually, 35 patients were eligible for the present analysis. Patient characteristics are displayed in Table 1. All tumors were staged according to the 2002 Union for International Cancer Control Tumor, Node, Metastasis staging system.

\section{Treatment and Follow-Up}

All patients underwent concurrent chemoradiotherapy. External radiation therapy was administered in 2-Gy daily standard fractions by using 4-MV x-ray, and CT-based 3D conformal radiation therapy was mandatory. The gross tumor volume and the bulky lymph nodes were treated with up to 60-70 Gy (median, 68.4 Gy). A prophylactic nodal area was irradiated with up to 40-50 Gy (median, $44.6 \mathrm{~Gy}$ ). Patients received concurrent chemotherapy by using S-1 and cisplatin: S-1 at the dose of $60 \mathrm{mg} / \mathrm{m}^{2}$ for 3 weeks followed by 1 week of rest plus weekly cisplatin at the dose of 30 $\mathrm{mg} / \mathrm{m}^{2}$ for 3 weeks followed by 1 week of rest $(n=25)$ or cisplatin, $100 \mathrm{mg} / \mathrm{m}^{2}$, at weeks 1 and $4(n=10)$. Chemotherapy was repeated every 4 weeks for 2 courses.

Pretreatment diagnostic examinations included contrast-enhanced CT in all patients, $\left[{ }^{18} \mathrm{~F}\right] \mathrm{FDG}-\mathrm{PET} / \mathrm{CT}$ in 20 patients, and panendoscopy with biopsy in all patients. For routine pretreatment examinations, MR imaging with DWI was performed in all patients. Pretreatment MR imaging with DWI was performed from 1 to 10 days before the start of treatment, and a second MR imaging with DWI was performed at 3 weeks after the start of treatment. In the previous study of the usefulness of DWI in predicting the response to neoadjuvant chemoradiotherapy for 


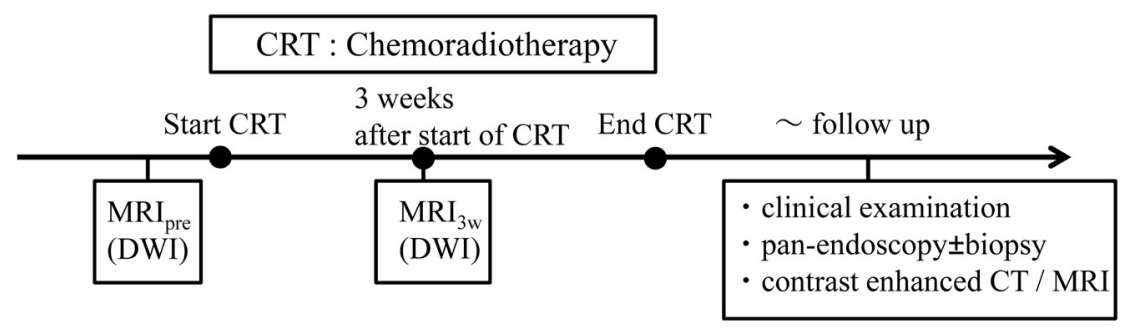

Remission $\rightarrow$ locoregional control: continue follow-up

Lesion progression $\rightarrow$ biopsy $(+)$ and/or increase of lesion size : locoregional failure biopsy $(-) \rightarrow$ continue follow up

FIG 2. Time line illustrating the consecutive methodologic steps in this study.
Imaging Analysis. All primary tumors and lymph nodes diagnosed as metastatic nodes on pretreatment clinical and imaging assessment were evaluated on MR imaging with DWI performed from 1 to 10 days before the start of treatment and MR imaging with DWI performed at 3 weeks after the start of treatment, respectively. Because the images of HNSCC are subject to artifacts induced by continuous physiologic motion such as breathing and swallowing as well as susceptibility artifacts, automated evaluation of serial changes in ADC, such as a histogram-based or voxel-
HNSCC, a significant ADC change to predict the treatment response was obtained at a cumulative dose of $30 \mathrm{~Gy} .{ }^{11}$ Therefore, in this study, the second MR imaging with DWI was performed at 3 weeks after the start of treatment, reaching a cumulative dose of $30 \mathrm{~Gy}$ to the primary tumors and metastatic nodes. After the treatment, patients were followed to evaluate locoregional control (LRC) by clinical examination and a panendoscopy, followed by biopsy in cases of suspected residual disease.

In addition, routine follow-up contrast-enhanced CT and MR imaging were performed every 6 months during the follow-up period. The follow-up period was designated as the total time of follow-up starting at treatment completion and ending with either tumor recurrence or at the last patient contact without tumor recurrence. Tumor recurrence was defined at primary and nodal sites by a persistent or recurrent mass with either histopathologic proof or an increase in lesion size on serial CT or MR imaging examinations. A time line describing the consecutive methodologic steps in this study is shown in Fig 2. The progression-free survival period was defined as the time between assignment and disease progression, death, or last known follow-up. The median follow-up period for all patients was 30.8 months (range, 7-56 months); the median follow-up in survivors (no evidence of disease) was 37.6 months (range, 24-56 months).

\section{MR Imaging and Imaging Analysis}

Diffusion-Weighted MR Imaging. MR imaging was performed by using a 1.5T system (Avanto; Siemens, Erlangen, Germany) with a neck coil or a neurovascular coil. All sequences extended from the skull base to the thoracic outlet. The imaging protocol consisted of the following: T2-weighted axial and coronal images acquired by using a turbo spin-echo sequence (TR/TE $=4000 / 90 \mathrm{~ms}$, $512 \times 256$ matrix), and T1-weighted axial images acquired by using a gradient recalled-echo sequence $(\mathrm{TR} / \mathrm{TE}=630 / 12 \mathrm{~ms}$, $512 \times 256$ matrix). DWI was performed with a single-shot spinecho echo-planar imaging sequence by using a short inversion recovery time for fat suppression $(\mathrm{TR} / \mathrm{TE} / \mathrm{TI}=4000 / 68 / 180 \mathrm{~ms}$, $512 \times 256$ matrix). The sequence was repeated for 3 values of the motion-probing gradients $\left(b=0,90\right.$, and $\left.800 \mathrm{~s} / \mathrm{mm}^{2}\right)$. The motion-probing gradients were placed on the 3 directions with the same strength. The FOV was $25 \mathrm{~cm}$, and the section thickness was $6 \mathrm{~mm}$ with an intersection gap of $3 \mathrm{~mm}$. The ADC map was reconstructed for each pixel by the b-values of 90 and $800 \mathrm{~s} / \mathrm{mm}^{2}$ by using the standard software on the console (syngo; Siemens). wise approach incorporating registered image datasets between treatment interval examinations, may be needed to decrease interpretation error. Therefore, in this study, the mean value of $\mathrm{ADC}$ of the whole tumor and the mean change in ADC during treatment were used. ROIs were independently placed over all targeted lesions on every section of the ADC map, and the ADC values for the sections were averaged to obtain the mean value of $\mathrm{ADC}$ of the whole tumor for each of the patients at each measurement time point. For region-of-interest placements in the lesions, care was taken to include the solid portions of the lesions and to exclude any obviously cystic or necrotic areas in reference to the T2WI. In addition, these ROIs were used to measure the whole tumor volume. In each primary tumor and metastatic node, whole tumor volume was calculated by multiplying each crosssectional area by the section thickness. These procedures were performed separately and independently by 2 observers (M.M. and Y.K., with 15 and 20 years of experience in head and neck MR imaging, respectively) who were blinded to the information regarding local failure or control. All ADC and tumor volume measurements were performed twice by each observer.

The $\triangle \mathrm{ADC}$ at 3 weeks for each primary tumor and metastatic node was calculated on the basis of the ADC values at pretreatment and 3 weeks after the start of treatment by using the formula

$$
\Delta \mathrm{ADC}=\left(\mathrm{ADC}_{3 \mathrm{~W}} \cdot \Delta \mathrm{ADC}_{\mathrm{pre}}\right) / \mathrm{ADC}_{\mathrm{pre}},
$$

where $A D C_{\text {pre }}$ represents the pretreatment $\mathrm{ADC}$ values and $\mathrm{ADC}_{3 \mathrm{~W}}$ represents the $\mathrm{ADC}$ values at 3 weeks after the start of treatment.

In addition, the tumor regression rate $(\Delta \mathrm{TV})$ for each primary tumor and metastatic node was calculated on the basis of the tumor volume at pretreatment and 3 weeks after the start of treatment by using the formula

$$
\Delta \mathrm{TV}=\left(\mathrm{TV}_{\mathrm{pre}}-\mathrm{TV}_{3 \mathrm{~W}}\right) / \mathrm{TV}_{\mathrm{pre}},
$$

where $\mathrm{TV}_{\text {pre }}$ represents the pretreatment tumor volume and $\mathrm{TV}_{3 \mathrm{~W}}$ represents the tumor volume at 3 weeks after the start of treatment.

\section{Statistical Analysis}

The intraobserver and interobserver variability of region-of-interest placement for the measurement of ADC and tumor volume of primary tumors and metastatic nodes was analyzed by calculating the interclass correlation coefficient for single measurements $(0-0.20$ is considered poor; $0.21-0.40$, as fair; $0.41-0.60$, 

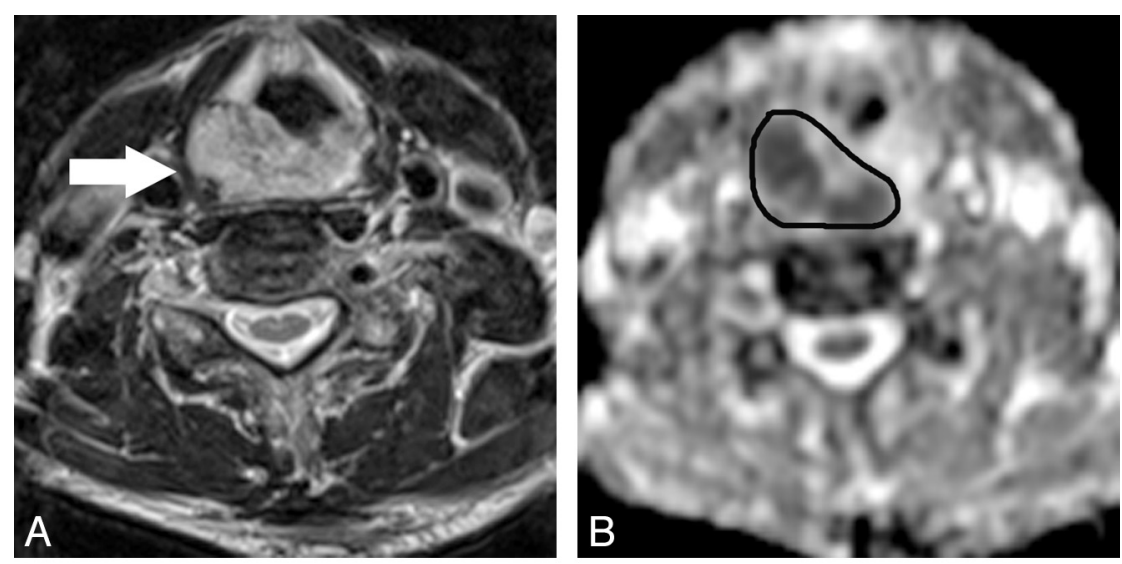

Statistical calculations were performed by using statistical analysis software (Statistical Package for the Social Sciences, Version 15.0; IBM, Armonk, New York), and $P$ values $<.05$ were considered statistically significant.

\section{RESULTS}

\section{Treatment Outcome}

During the follow-up period, complete LRC was achieved in 21 of 35 patients (60\%). Four of 35 patients (11.4\%) developed an isolated local recurrence. Five of 35 patients $(14.3 \%)$ developed a regional recurrence without primary tumor recurrence. Five of 35 patients (14.3\%) developed a simultaneous locoregional tumor recurrence. Patients with locoregional recurrence were treated with salvage surgery and/or neck dissection with or without adjuvant chemotherapy. In patients whose tumors were inoperable, only chemotherapy was performed. Three of 35 patients (8.6\%) died during the follow-up period because of the extent of local recurrent tumor.

\section{Observer Agreement}

The intraobserver and interobserver agreement for the ADC measurement was 0.81 and 0.73 on primary tumors and 0.91 and 0.82 on metastatic nodes, respectively. The intraobserver and interobserver agreement for the tumor volume measurement was 0.80 and 0.70 on primary tumors and 0.92 and 0.83 on metastatic nodes, respectively.

as moderate; $0.61-0.80$, as good; and 0.81-1.00, as excellent correlation). The pretreatment $\mathrm{ADC} ; \triangle \mathrm{ADC}$; primary tumor volume; primary nodal volume; $\Delta \mathrm{TV}$; and the other clinical variables such as age, T stage (T1-2 versus T3-4), N stage (N0-1 versus N2-3), and tumor location (hypopharynx or oral cavity versus others) were compared with LRC and locoregional failure (LRF) by using a Mann-Whitney $U$ test. The univariate/multivariate nominal logistic analysis was used to assess the correlation between LRC and the same variables described above. Then, a receiver operating characteristic analysis with the area under the curve was used to investigate the discriminatory capability of the significant predictive value of LRC. For calculation of the sensitivity, specificity, and accuracy of the significant predictive value of LRC, the optimal threshold was determined by giving equal weighting to sensitivity and specificity on the receiver operating characteristic curve.

Finally, to determine the usefulness of $\triangle \mathrm{ADC}$ for the prediction of prognosis after chemoradiotherapy, we compared progression-free survival for the 2 groups divided by the optimal threshold value by using the Kaplan-Meier method followed by the log-rank test.

\section{Analysis of Variables for Treatment Response}

All DWI examinations of eligible patients for the present analysis were performed successfully. A representative case is shown in Fig 3.

Comparison of variables in LRC and LRF and univariate and multivariate analysis of variables in association with LRC are summarized in Table 2. The $\Delta \mathrm{ADC}_{\text {primary }}, \Delta \mathrm{ADC}_{\text {node }}$, primary tumor volume, primary nodal volume, $\Delta \mathrm{TV}_{\text {node }}, \mathrm{N}$ stage, and tumor location revealed significant differences between LRC and LRF; however, there was no significant difference in $\mathrm{ADC}_{\text {primary }}$, $\mathrm{ADC}_{\text {node }}, \Delta \mathrm{TV}_{\text {primary }}$, age, and $\mathrm{T}$ stage. In univariate logistic analysis, $\Delta \mathrm{ADC}_{\text {primary }}, \Delta \mathrm{ADC}_{\text {node }}, \Delta \mathrm{TV}_{\text {node }}, \mathrm{N}$ stage, and tumor location showed significant association with LRC. Primary tumor volume and primary nodal volume showed no significant association. In multivariate logistic analysis after variable selection with the use of the forward stepwise method, only $\triangle \mathrm{ADC}_{\text {primary }}$ was identified as a significant and independent predictor of LRC.

The receiver operating characteristic analysis resulted in a threshold $\Delta \mathrm{ADC}_{\text {primary }}$ of 0.24 and an area under the curve of 0.9 . 
Table 2: Comparison of variables in LRC and LRF/univariate and multivariate analysis of variables in association with LRC

\begin{tabular}{|c|c|c|c|c|c|c|c|c|c|}
\hline & \multicolumn{3}{|c|}{ Comparison of Variables } & \multicolumn{3}{|c|}{ Univariate Analysis } & \multicolumn{3}{|c|}{ Multivariate Analysis } \\
\hline & LRC & LRF & $P$ Value & $P$ Value & OR & $95 \% \mathrm{Cl}$ & $P$ Value & OR & $95 \% \mathrm{Cl}$ \\
\hline$A D C_{\text {primary }}$ & $1.18 \pm 0.29$ & $1.24 \pm 0.3$ & NS & NS & & & & & \\
\hline$A D C_{\text {node }}$ & $1.09 \pm 0.05$ & $1.15 \pm 0.03$ & NS & NS & & & & & \\
\hline$\triangle \mathrm{ADC}_{\text {primary }}$ & $0.6 \pm 0.31$ & $0.23 \pm 0.23$ & .0003 & .004 & $6.85 \times 10^{-4}$ & $0.48 \times 10^{-5}-0.1$ & .04 & $1.42 \times 10^{-3}$ & $0.21 \times 10^{-5}-0.94$ \\
\hline$\triangle \mathrm{ADC}_{\text {node }}$ & $0.47 \pm 0.11$ & $0.32 \pm 0.04$ & .01 & .03 & $1.78 \times 10^{-2}$ & $0.46 \times 10^{-3}-0.69$ & NS & & \\
\hline Primary tumor volume $\left(\mathrm{mm}^{3}\right)$ & $9,612 \pm 18,886$ & $32,122 \pm 65,012$ & .03 & NS & & & & & \\
\hline Primary nodal volume $\left(\mathrm{mm}^{3}\right)$ & $7,106 \pm 11,048$ & $4,788 \pm 6,999$ & .01 & NS & & & & & \\
\hline$\Delta \mathrm{TV}_{\text {primary }}$ & $0.72 \pm 0.14$ & $0.69 \pm 0.13$ & NS & NS & & & & & \\
\hline$\Delta T V_{\text {node }}$ & $0.71 \pm 0.19$ & $0.56 \pm 0.19$ & .02 & 002 & $1.23 \times 10^{-3}$ & $0.52 \times 10^{-5}-0.29$ & NS & & \\
\hline Age (yr) & $69.3 \pm 9.67$ & $66.9 \pm 12.6$ & NS & NS & & & & & \\
\hline $\mathrm{T}(\mathrm{T} 1-2$ vs T3-4) & $7 / 14$ & $8 / 6$ & NS & NS & & & & & \\
\hline$N(0-1$ vs $2-3)$ & $7 / 14$ & $3 / 11$ & .02 & .02 & 4.16 & $1.23-21.55$ & NS & & \\
\hline $\begin{array}{l}\text { Tumor location (hypopharynx } \\
\text { or oral cavity vs others) }\end{array}$ & $4 / 17$ & $9 / 5$ & 003 & .03 & 0.22 & $0.05-0.88$ & NS & & \\
\hline
\end{tabular}

Note:-NS indicates a $P$ value $>.05$.

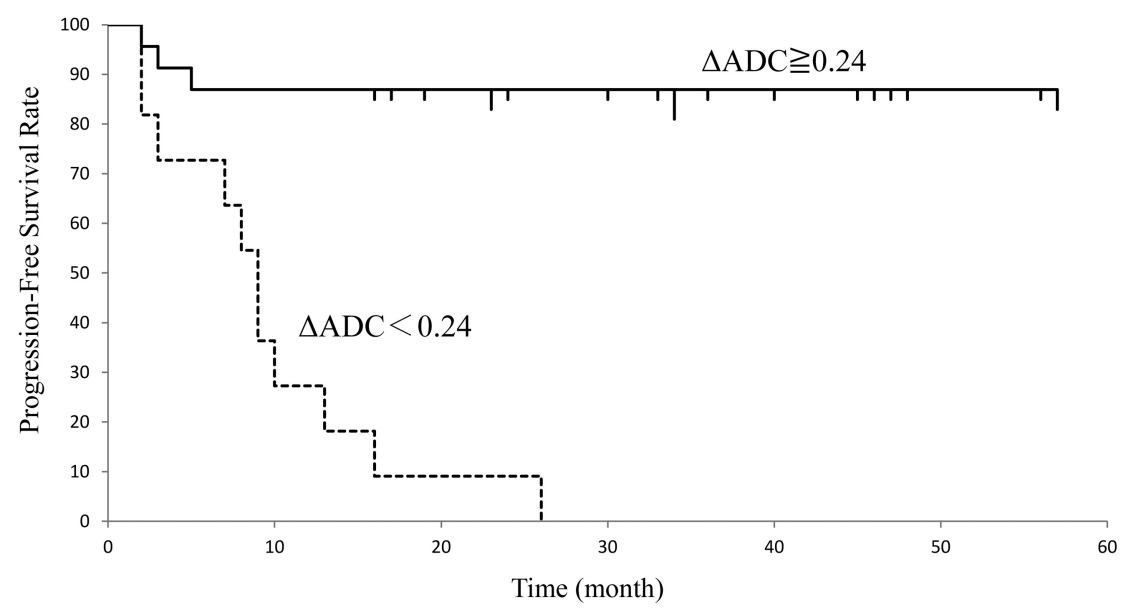

FIG 4. Progression-free survival of patients with head and neck squamous cell carcinoma assessed by $\triangle \mathrm{ADC}$. The graph shows that the median progression-free survival period of patients with $\triangle \mathrm{ADC} \geqq 0.24$ was significantly longer than that of patients with $\Delta \mathrm{ADC}<0.24(P<.05)$. cantly with the LRC and was more accurate than volumetric changes for the prediction of treatment outcome. In addition, King et $\mathrm{al}^{9}$ reported that a strong significant correlation was found between LRF and serial change in ADC. Thus, the optimal timing of the evaluation of ADC and its analysis method for predicting the treatment response to chemotherapy or chemoradiotherapy in HNSCC have not been established.

In the current study, the $\triangle \mathrm{ADC}_{\text {primary }}$ at 3 weeks of treatment was significantly lower for lesions with LRF than for those with LRC, and in the multivariate analysis, only $\triangle \mathrm{ADC}_{\text {primary }}$ revealed a significant association with LRC. By contrast, pretreatment $\mathrm{ADC}_{\text {primary }}$ was not statistically correlated with LRC. In addition, the

The 2-by-2 contingency table based on a $\Delta \mathrm{ADC}_{\text {primary }}$ of 0.24 revealed a sensitivity of $100 \%$, specificity of $78.7 \%$, positive predictive value of $76.7 \%$, negative predictive value of $100 \%$, and overall accuracy of $84.8 \%$ for the prediction of LRC.

The progression-free survival curves in patients with $\Delta \mathrm{ADC}_{\text {primary }} \geq 0.24$ and $\Delta \mathrm{ADC}_{\text {primary }}<0.24$ are shown in Fig 4 . The median progression-free survival period of patients with $\Delta \mathrm{ADC}_{\text {primary }}<0.24$ was $11.5 \pm 7.6$ and that of patients with $\Delta \mathrm{ADC}_{\text {primary }} \geq 0.24$ was $32.8 \pm 8.2$. The difference in progression-free survival between the 2 groups divided by the threshold value of $\triangle \mathrm{ADC}_{\text {primary }}$ was significant $(P<.05)$.

\section{DISCUSSION}

In previous clinical studies evaluating the use of DWI to predict treatment response to radiation therapy or chemoradiotherapy in HNSCC, 2 ADC parameters-namely, pretreatment ADC and the change in ADC during or early after treatment-have been shown to be useful. Kim et $\mathrm{al}^{6}$ reported the usefulness of pretreatment ADC for predicting the treatment response of neck lymph nodes at the end of treatment. In addition, Hatakenaka et $\mathrm{al}^{7}$ reported the usefulness of pretreatment ADC for predicting local failure during follow-up after chemoradiotherapy or radiation therapy. On the other hand, Vandecaveye et $\mathrm{al}^{8}$ reported that the change in ADC at 2 and 4 weeks of treatment correlated signifi-
$\Delta \mathrm{ADC}_{\text {primary }}$ threshold value of 0.24 resulted in $100 \%$ sensitivity and $100 \%$ negative predictive value for the prediction of LRC. The high negative predictive value of $\triangle \mathrm{ADC}_{\text {primary }}$ may help to predict patients with LRF of chemoradiotherapy at the early phase of treatment. Furthermore, in comparison of progression-free survival by using the $\triangle \mathrm{ADC}_{\text {primary }}$ threshold value of 0.24 for distinguishing the LRC group from the LRF group, the patients with $\Delta \mathrm{ADC}_{\text {primary }} \geq 0.24$ showed better prognosis than those with $\triangle \mathrm{ADC}_{\text {primary }}<0.24$. Therefore, our results indicate that $\Delta \mathrm{ADC}_{\text {primary }}$ is a potential predictive indicator of treatment response to chemoradiotherapy but that pretreatment $\mathrm{ADC}_{\text {primary }}$ is not. However, further studies that prospectively use the thresholds obtained in this study are necessary to determine the real significance of $\triangle \mathrm{ADC}_{\text {primary }}$ for prediction and management of patients with HNSCC treated with chemoradiotherapy.

In many previous clinical and animal model studies, tumors showing a rise in the ADC at an early phase of treatment showed a better treatment response than those with little or no ADC rise. ${ }^{12,13}$ Although the mechanism of rise in the ADC at an early phase of treatment following cytotoxic and radiation treatment in experimental and human tumors is not fully understood, it has been speculated that a rise in ADC might be attributed to an increase in the fractional volume and diffusion of water molecules in the extracellular space that occurs with the disorganized micro- 
structure in necrosis and apoptosis in response to treatment. ${ }^{14}$ Therefore, $\triangle \mathrm{ADC}$ at an early phase of treatment seems to reflect the degree of tumor cell damage resulting from the treatment. However, because the treatment response may be attributed to differences in tumor aggressiveness, the treatment method, or the intensity of treatment, the use of only a single ADC measurement at pretreatment appears to be inadequate for the prediction of treatment response. Therefore, evaluating the $\triangle \mathrm{ADC}$ may be necessary for the prediction of treatment response.

The induction of tiny regions of liquefaction necrosis at the early phase of treatment may interfere with ADC measurement. ${ }^{15}$ For this reason, there may be a misleading and misrepresentative rise in $\mathrm{ADC}$ despite the persistence of viable tumor components. In the ADC measurement in our study, it may have been difficult to distinguish tiny liquefaction necrosis from lesions at 3 weeks of treatment because the lesions were visually associated on the ADC map in reference to T2WI for tumor heterogeneity. Therefore, we used the mean value of ADC of the whole tumor and the mean change in ADC during treatment. The use of the mean change in ADC may be explained by the fact that the specificity and positive predictive value of $\triangle \mathrm{ADC}$ were low in the current study. In brain tumor, quantification of diffusion changes has evolved from the mean change in ADC to a voxel-by-voxel approach, termed the "functional diffusion map," as a biomarker for treatment response. ${ }^{16}$ In the functional diffusion map, treatment response is evaluated on the basis of the fractional volume of significantly increased ADC within tumor. In the study by Galbán et al ${ }^{17}$ of the functional diffusion map of HNSCC, the change in ADC assessed by the functional diffusion map was superior to the percentage change of the mean ADC in prediction of disease control after chemoradiotherapy. Therefore, in the future, more automated evaluations, such as a voxel-by-voxel approach, may be needed to estimate the change in ADC during treatment more accurately. However, it may be difficult to implement it in routine examinations for organs outside the brain due to differences in the orientation of images and artifacts induced by continuous physiologic motion.

In many previous studies that used DWI to examine the treatment response in HNSCC, a maximum b-value of $1000 \mathrm{~s} / \mathrm{mm}^{2}$ was used..$^{6-10}$ Preferentially, a standardized ADC calculation by using at least $3 \mathrm{~b}$-values, including a maximum b-value exceeding $500 \mathrm{~s} / \mathrm{mm}^{2}$, should be performed. ${ }^{18}$ In the current study, a maximum b-value of $800 \mathrm{~s} / \mathrm{mm}^{2}$ was used to limit the possible effects of distortion due to susceptibility artifacts and to reduce the signal-to-noise ratio on the ADC value; such factors are problems at high b-values. In this study, only 2 patients were excluded from this study due to a low signal-to-noise ratio or artifacts of DWI. The merit of ADC values differs with b-values because they are influenced by tissue perfusion and $\mathrm{T} 2$ time, and it may be desirable for accurate ADC measurement that 1 of the $b$ values not be zero. Therefore, in this study, ADC values were calculated from b-values of 90 and $800 \mathrm{~s} / \mathrm{mm}^{2}$, and DWI with a b-value of zero was used for image registration.

With regard to the relationship between the ADC of metastatic nodes and treatment response, Kim et $\mathrm{al}^{6}$ reported that the change in ADC of metastatic nodes within the first week of chemoradiotherapy was more useful for predicting treatment response than pretreatment ADC. In addition, Vandecaveye et $\mathrm{al}^{8}$ reported that the change in the ADC of metastatic nodes at 2 and 4 weeks after the start of treatment correlated significantly with 2-year LRC. In this study, $\triangle \mathrm{ADC}_{\text {node }}$ revealed a significant difference between LRC and LRF and showed a significant association with LRC in univariate analysis. Therefore, our results were comparable with theirs, and it was suggested that the change in ADC of metastatic nodes during treatment may be useful for the prediction of treatment response and/or LRC. The primary sites of HNSCC are generally located at the air-tissue interface and in areas prone to motion artifacts induced by physiologic motion such as breathing and swallowing. Therefore, in DWI, the primary sites seem to be more influenced by physiologic motion and susceptibility artifacts than cervical lymph nodes. In addition, in variability analysis of region-of-interest placement for measurements of ADC, intraobserver and interobserver agreement of the metastatic nodes tended to be higher than those of the primary tumors in this study. Therefore, although only the $\triangle \mathrm{ADC}_{\text {primary }}$ was identified as a significant and independent predictor of LRC in this study, the possibility that ADC values from the metastatic nodes may predict LRC in patients with HNSCC treated with chemoradiotherapy was thought to have great clinical significance.

The value of the primary tumor volume and $\mathrm{T}$ stage as a prognostic factor in HNSCC has been reported in published studies for multiple subsites and different treatment modalities. ${ }^{7,19}$ However, in the current study, primary tumor volume and T stage did not show a significant correlation with LRC. Most previously published studies included patients treated with single-technique therapy (radiation therapy or surgery alone). However, in this study, all patients were treated with definitive concurrent chemoradiotherapy. There have been many reports that definitive concurrent chemoradiotherapy leads to better clinical outcome than single-technique therapy in HNSCC. ${ }^{20}$ Therefore, it was speculated that clinical outcome after definitive concurrent chemoradiotherapy might not be significantly influenced by primary tumor volume or T stage.

In patients with HNSCC treated with chemoradiotherapy, controversies remain concerning the role of neck dissection for the management of the neck with bulky lymph node involvement. ${ }^{21}$ There is no consensus on the treatment of patients with a complete regional response after treatment. With regard to the regional recurrence after chemoradiotherapy, it has been reported that lymph node residual size and the regression rate of nodal maximal diameter or nodal volume after treatment might be useful for the prediction of regional recurrence. ${ }^{22,23}$ In this study, we evaluated the usefulness of the tumor regression ratio at 3 weeks after the start of chemoradiotherapy for prediction of LRC. As a result, $\Delta \mathrm{TV}_{\text {node }}$ revealed a significant difference between LRC and LRF and showed significant association with LRC in univariate analysis. Therefore, if prediction of regional recurrence is possible by the tumor regression rate of metastatic nodes during treatment, it has great clinical significance. In the future, it would be interesting to evaluate whether $\Delta \mathrm{TV}_{\text {node }}$ may be a useful criterion to guide clinical decisions regarding neck dissection after chemoradiotherapy.

There are limitations to our study. First, the patient population was relatively small and heterogeneous, including those with 
tumors from various head and neck sites. Also in this study, patients with oral cavity cancer were included. Surgery is usually the preferred treatment option in patients with oral cavity carcinoma, but these patients whose disease was considered inoperable because of tumor extent and/or medical reasons were enrolled in this study. Therefore, further studies with a large number of patients without potential selection bias are needed because direct comparison among DWI and other predictive or prognostic factors is necessary to show the actual clinical significance of our findings. Second, the biologic differences in squamous cell carcinomas due to differences in smoking and alcohol use as well as molecular markers such as epidermal growth factor receptor expression and human papillomavirus infection have been suggested as prognostic factors. ${ }^{24}$ In particular, human papillomavirus-positive oropharyngeal carcinoma has emerged as a new entity with an excellent overall survival rate, but the patients in this study were not tested for human papillomavirus infection.

\section{CONCLUSIONS}

DWI provides information that may be used as a predictive imaging biomarker of LRC in patients with HNSCC treated by chemoradiotherapy. The $\triangle \mathrm{ADC}_{\text {primary }}$ at 3 weeks during treatment is a valid predictive clinical factor, showing a significant association with LRC. Thus, sequential DWI may help to avoid ineffective treatment and unnecessary toxicity, allowing chemoradiotherapy to be selectively used for appropriate patients.

\section{REFERENCES}

1. Argiris A, Karamouzis MV, Raben D, et al. Head and neck cancer. Lancet 2008;371:1695-709

2. Horiot JC, Bontemps P, van den Bogaert W, et al. Accelerated fractionation (AF) compared with conventional fractionation (CF) improves locoregional control in the radiotherapy of advanced head and neck cancers: results of the EORTC 22851 randomized trial. Radiother Oncol 1997;44:111-21

3. Ang KK, Tortti A, Brown BW, et al. Randomized trial addressing risk feature and time factors of surgery plus radiotherapy in advanced head-and-neck cancer. Int J Radiat Oncol Biol Phys 2001;51:571-78

4. Chiesa F, Mauri S, Tradati N, et al. Surfing prognostic factors in head and neck cancer at the millennium. Oral Oncol 1999;35:590-96

5. Chenevert TL, Stegman LD, Taylor JM, et al. Diffusion magnetic resonance imaging: an early surrogate marker of therapeutic efficacy in brain tumors. J Natl Cancer Inst 2000;92:2029-36

6. Kim S, Loevner L, Quon H, et al. Diffusion-weighted magnetic resonance imaging for predicting and detecting early response to chemoradiation therapy of squamous cell carcinomas of the head and neck. Clin Cancer Res 2009;15:986-94

7. Hatakenaka M, Nakamura K, Yabuuchi H, et al. Pretreatment apparent diffusion coefficient of the primary lesion correlates with local failure in head-and-neck cancer treated with chemoradiotherapy or radiotherapy. Int J Radiat Oncol Biol Phys 2011;81:339-45

8. Vandecaveye V, Dirix P, De Keyzer F, et al. Predictive value of diffusion-weighted magnetic resonance imaging during chemoradio- therapy for head and neck squamous cell carcinoma. Eur Radiol 2010;20:1703-14

9. King AD, Mo FK, Yu KH, et al. Squamous cell carcinoma of the head and neck: diffusion-weighted MR imaging for prediction and monitoring of treatment response. Eur Radiol 2010;20:2213-20

10. Vandecaveye V, Dirix P, Keyzer FD, et al. Diffusion-weighted magnetic resonance imaging early after chemoradiotherapy to monitor treatment response in head-and-neck squamous cell carcinoma. Int J Radiat Oncol Biol Phys 2012;82:1098-107

11. Kato $H$, Kanematsu M, Tanaka $O$, et al. Head and neck squamous cell carcinoma: usefulness of diffusion-weighted MR imaging in the prediction of a neoadjuvant therapeutic effect. Eur Radiol 2009;19:103-09

12. Hamstra DA, Rehemtulla A, Ross BD. Diffusion magnetic resonance imaging: a biomarker for treatment response in oncology. J Clin Oncol 2007;25:4104-09

13. Roth Y, Tichler T, Kostenich G, et al. High-b-value diffusionweighted MR imaging for pretreatment prediction and early monitoring of tumor response to therapy in mice. Radiology 2004; 232:685-92

14. Hamstra DA, Lee KC, Moffat BA, et al. Diffusion magnetic resonance imaging: an imaging treatment response biomarker to chemoradiotheray in a mouse model of squamous cell cancer of head and neck. Transl Oncol 2008;1:187-94

15. Padhani AR, Liu G, Koh DM, et al. Diffusion-weighted magnetic resonance imaging as a cancer biomarker: consensus and recommendations. Neoplasia 2009;11:102-25

16. Moffat BA, Chenevert TL, Lawrence TS, et al. Functional diffusion map: a noninvasive MRI biomarker for early stratification of clinical brain tumor response. Proc Natl Acad Sci US A 2005;102:5524-29

17. Galbán CJ, Mukherji SK, Chenevert TL, et al. A feasibility study of parametric response map analysis of diffusion-weighted magnetic resonance imaging scans of head and neck cancer patients for providing early detection of therapeutic efficacy. Transl Oncol 2009;2:184-90

18. Koh DM, Collins DJ. Diffusion-weighted MRI in the body: applications and challenges in oncology. AJR Am J Roentgenol 2007; 188:1622-35

19. Chung EJ, Lee NJ, Baek SK, et al. Clinical efficacy of primary tumor volume measurements: comparison of different primary sites. Clin Exp Otorhinolaryngol 2009;2:78-84

20. Pignon JP, Bourhis J, Designe L. Chemotherapy added to locoregional treatment for head and neck squamous-cell carcinoma: three meta-analyses of updated individual data. Lancet 2000; 355:949-55

21. Lango MN, Myers JN, Garden AS. Controversies in surgical management of the node-positive neck after chemoradiation. Semin Radiat Oncol 2009;19:24-28

22. Labadie RF, Yarbrough WG, Weissler MC, et al. Nodal volume reduction after concurrent chemo- and radiotherapy: correlation between initial CT and histopathologic findings. AJNR Am J Neuroradiol 2000;21:310-14

23. Clavel S, Charron MP, Belair M, et al. The role of computed tomography in the management of the neck after chemoradiotherapy in patients with head-and-neck cancer. Int J Radiat Oncol Biol Phys 2012;82:567-73

24. Fakhry C, Westra WH, Li S, et al. Improved survival of patients with human papillomavirus: positive head and neck squamous cell carcinoma in a prospective clinical trial. J Natl Cancer Inst 2008;100: 261-69 